\title{
Maternal Immune Activation Causes Behavioral Impairments and Altered Cerebellar Cytokine and Synaptic Protein Expression
}

\author{
Gurudutt Pendyala', ${ }^{1,3}$ Shinnyi Chou', Yoosun Jung', Pierluca Coiro', Elizabeth Spartz', \\ Ragunathan Padmashri', Ming $\mathbf{L i}^{2}$ and Anna Dunaevsky*,I \\ 'Department of Developmental Neuroscience, Munroe-Meyer Institute, University of Nebraska Medical Center, Omaha, NE, USA; ${ }^{2}$ Department of \\ Psychology, University of Nebraska -Lincoln, Lincoln, NE, USA
}

\begin{abstract}
Emerging epidemiology studies indicate that maternal immune activation (MIA) resulting from inflammatory stimuli such as viral or bacterial infections during pregnancy serves as a risk factor for multiple neurodevelopmental disorders including autism spectrum disorders and schizophrenia. Although alterations in the cortex and hippocampus of MIA offspring have been described, less evidence exists on the impact on the cerebellum. Here, we report altered expression of cytokines and chemokines in the cerebellum of MIA offspring, including increase in the neuroinflammatory cytokine TNF $\alpha$ and its receptor TNFRI. We also report reduced expression of the synaptic organizing proteins cerebellin-I and GluR $\delta 2$. These synaptic protein alterations are associated with a deficit in the ability of cerebellar neurons to form synapses and an increased number of dendritic spines that are not in contact with a presynaptic terminal. These impairments are likely contributing to the behavioral deficits in the MIA exposed offspring.

Neuropsychopharmacology (2017) 42, |435-1446; doi:I0.1038/npp.2017.7; published online I5 February 2017
\end{abstract}

\section{INTRODUCTION}

Epidemiology studies indicate that maternal immune activation (MIA) resulting from inflammatory stimuli, viral or bacterial infections of pregnant mothers is a risk factor for multiple neurodevelopmental disorders such as autism spectrum disorder (ASD), and schizophrenia (SZ) as well as other neuropsychiatric disorders (Knuesel et al, 2014). Since the genetic contribution to both ASD and SZ is well documented it is likely that a convergence of genetic alterations and environmental insult during early development lead to the development of these disorders (Atladottir et al, 2010; Patterson, 2011).

Evidence points to immunological dysfunction in ASD that may result in inflammatory-like state with MIA. Specifically, altered expression of cytokines and markers of oxidative stress as well as presence of activated astrocytes and microglia has been reported in brains and cerebrospinal fluid obtained from young and old individuals with ASD (Ashwood et al, 2011a; Chez et al, 2007; Morgan et al, 2010; Patterson, 2009, 2011; Sajdel-Sulkowska et al, 2011; Vargas

\footnotetext{
*Correspondence: Dr A Dunaevsky, Department of Developmental Neuroscience, Munroe-Meyer Institute, University of Nebraska Medical Center, 985960 Nebraska Medical Center, Omaha, NE 68198-5960, USA, Tel: +402559 I07I, E-mail: adunaevsky@unmc.edu

${ }^{3}$ Current address: Department of Anesthesiology, University of Nebraska Medical Center, Omaha, NE 68198, USA

Received 23 February 2016; revised 21 December 2016; accepted 2 January 2017; accepted article preview online 19 January 2017
}

et al, 2005; Wei et al, 2011). The chronic neuroinflammation is thought to contribute to the symptoms and pathology of $\mathrm{ASD}$, such as altered neuronal connectivity and function (Coiro et al, 2015; Hutsler and Zhang, 2010; Patrich et al, 2016; Penzes et al, 2011).

Animal models, including both rodents and non-human primates with MIA during gestation demonstrate that offspring have altered behavioral phenotypes relevant to ASD and SZ including impaired ultrasonic vocalizations, social interactions and repetitive behaviors (Choi et al, 2016; Malkova et al, 2012; Patterson, 2009; Schwartzer et al, 2013). In addition to the behavioral impairments, MIA offspring also exhibit changes in peripheral immune cells (Onore et al, 2014) as well as changes in the central nervous system. Specifically, alterations in cytokine and chemokine levels during and beyond the period of synaptogenesis have been reported in the cortex and the hippocampus of MIA offspring in some studies (Bauman et al, 2014; Borrell et al, 2002; Ehninger et al, 2012; Fatemi et al, 2002; Malkova et al, 2012; McAlonan et al, 2010; Shi et al, 2003). Moreover, impairments in cortical and hippocampal synaptic development and function have been observed on neurons from young and adult MIA offspring (Coiro et al, 2015; Elmer et al, 2013; Ito et al, 2010; Patrich et al, 2016).

The cerebellum is one of the brain regions most consistently seen to be affected in ASD (Courchesne et al, 1988) with functional cerebellar impairments observed in both ASD and SZ (Allen and Courchesne, 2003; Brown et al, 2005; Pierce and Courchesne, 2001). Although the number of 
Purkinje neurons observed in MIA offspring has been shown to be reduced (Shi et al, 2009), it is not known if cytokines are altered in the cerebellum of MIA offspring and whether cerebellar synapses are impaired. Here we used one of the most characterized mouse models of MIA; by eliciting a maternal anti-viral inflammatory response using a viral mimic, the synthetic dsRNA, poly(I:C) which activates the toll-like receptor (TLR) 3 (Patterson, 2009, 2011). In the current study, we demonstrate alterations in the expression of chemokines and cytokines in the cerebellum of MIA offspring during the course of synaptogenesis. We further demonstrate that the MIA offspring display synaptic and behavioral deficits. Altogether, these data indicate that MIA leads to chronic changes in the cerebellum and that altered cerebellar synaptic development might contribute to impaired behavior.

\section{MATERIALS AND METHODS}

\section{Ethics}

Mice were cared for in accordance with NIH guidelines for laboratory animal welfare. All experiments were approved by the University of Nebraska Medical Center Institutional Animal Care and Use Committee.

\section{Animals}

Transgenic FVB/N Tg(Pcp2-EGFP)BT153Gsat/Mmmh mice were obtained from GENSAT. All animals were housed in standard polycarbonate cages in a room under controlled $12: 12 \mathrm{~h}$ light/dark cycle, temperature and had ad libitum food and water access.

\section{Breeding}

The breeding cage comprised of two females and a male. Successful mating was confirmed next day with the presence of vaginal plug, and that day was referred to E0. Once a pregnant female was identified, it was removed from the breeding cage and housed individually in a standard cage.

\section{Induction of MIA}

MIA was induced using the viral mimic poly(I:C) as described in an earlier study (Garay et al, 2013). Briefly, pregnant mice were injected intraperitoneally (i.p.) on E12.5 with saline or poly(I:C) potassium salt (Sigma Aldrich; St Louis, MO). E12.5 was chosen since this stage of gestation correlates with the late first trimester in humans (Clancy et $a l, 2007)$ the time that infections are most closely linked to increased incidence of SZ and ASD (Atladottir et al, 2010). Poly(I:C) was freshly dissolved in saline and administered $i$. p. at $20 \mathrm{mg} / \mathrm{kg}$ based on the weight of the poly(I:C) itself, not including the total weight of the potassium salts. Control mice were injected with saline alone. This concentration of poly(I:C) is higher than that used for intravenous injections (Meyer et al, 2006) and was selected because it is the optimal i.p. dose that causes MIA, while preserving viability of offspring (Ito et al, 2010). Pups remained with the mother until weaning on postnatal day (P) 21 , at which time mice were group housed.
Behavioral tests. All behavioral tests were performed with the observer blinded to the experimental conditions. 5-9 litters per condition were used in the behavioral studies including both male and female offspring.

\section{Ultrasonic Vocalization}

USV testing was performed on postnatal days $6,8,10,12$, and 14. On these days mothers were removed from the home cage and placed in a separate room away from the pups. The pups remained in the home cage without mothers for 15 min. Immediately following maternal separation, both male and female pups were placed individually into plastic bowls (14.5 and $9.0 \mathrm{~cm}$ wide at the top and bottom, $7.5 \mathrm{~cm}$ high). Each bowl was then placed in a sound-attenuated chamber $(96.52 \mathrm{~W} \times 35.56 \mathrm{D} \times 63.5 \mathrm{~cm} \mathrm{H}$, Med Associated, St Albans, VT) equipped with an ultrasonic vocalization microphone (P48 Avisoft Bioacoustics/Emkay Microphone, Avisoft Bioacoustics, Berlin, Germany) mounted on the ceiling. The microphone was connected via an E-MU 0404 USB Audio device to a computer. Acoustic data were displayed in real time by the Avisoft RECORDER, a multichannel triggering hard-disk recording software (version 3.4; Avisoft Bioacoustics), and were recorded at a sampling rate of $192 \mathrm{kHz}$ in 16 bit format and analyzed by Avisoft SASLab Pro (version 4.51; Avisoft Bioacoustics).

\section{Marble Burying}

Marble burying was performed on P40 animals from the two groups. To a $38.5 \times 30 \mathrm{~cm}$ mouse cage containing $3.5 \mathrm{~cm}$ of corn cob bedding (277.4 g/l, GREENPRODUCTS, IA, USA) $200 \mathrm{~g}$ of Tek-fresh bedding (80.3 g/l, Harlan, IN, USA) was added and mixed thoroughly. Mice were acclimatized in such a cage for $24 \mathrm{~h}$ before testing. On the day of testing a similar new cage was setup and each mouse was acclimatized to it for $30 \mathrm{~min}$ prior to testing. Bedding was remixed immediately prior to testing and 30 glass marbles were placed on the bedding at a $5 \times 6$ arrangement. Individual mice were placed in the cage with the lid closed for $30 \mathrm{~min}$. The mouse was then removed and marbles were scored. A marble was considered buried if $2 / 3$ of it was covered by bedding.

\section{Rotarod}

Mice (6 weeks old) were placed on the motorized rod in the chamber (Rotamex 4/8, Columbus Instruments). Rotarod testing consisted of three trials per day ( $10 \mathrm{~min}$ inter-trial interval) over the course of 4 days. Acclimatization was carried out at a constant speed (4 r.p.m. over $180 \mathrm{~s}$ on days 1 and 2; 8 r.p.m. over $180 \mathrm{~s}$ on days 3 and 4 ) before training. In the first phase of the testing (days 1 and 2), the rotation speed gradually increased from 4 to 40 r.p.m. over a course of 300 s. In the second phase of testing (days 3 and 4), the rotation speed gradually increased from 8 to 79 r.p.m. over a course of $300 \mathrm{~s}$. The time latency was recorded when a mouse fell off, made one complete backward revolution while hanging on or reached $300 \mathrm{~s}$ on the rod. 


\section{Social Interactions}

Both male and female offspring were tested for social interaction in adulthood (after postnatal day 90). Individual animals were first habituated for 2 days in the testing chamber. One day post habituation, same sex animals from the same prenatal treatment condition were paired and placed in the testing chamber simultaneously, and their behaviors were video recorded for $5 \mathrm{~min}$. Animals were then returned to the home cage for $10 \mathrm{~min}$, and the procedure was repeated two more times, resulting in three 5-min sessions with the same test partner. In a fourth 5-min recording session, animals were paired with a novel partner from the same prenatal treatment condition.

The amount of time that a pair of animals engaged in social behaviors, regardless of the initiating subject, were determined by scorers blinded to the test conditions. Behaviors including sniffing, climbing over and crawling underneath partners, partner grooming, and partner pursuit were scored using the JWatcher behavioral coding software (http://www.jwatcher.ucla.edu/), and the total time spent in social behavior was used for analysis.

\section{Biochemical Assays}

Tissue lysates. Both male and female offspring from a total of 12 litters per condition were used. Brains were quickly snap frozen in liquid nitrogen and stored at $-80^{\circ} \mathrm{C}$ until further use. Tissues were lysed in homogenization buffer (50 mM Tris, $10 \mathrm{mM}$ EDTA, pH7.4) containing protease and phosphatase inhibitors using a small plastic pestle fitted to a motorized hand held homogenizer. Tissue was further triturated for complete homogenization. Tissue was incubated for $20 \mathrm{~min}$ in a cold room on a rotator and then centrifuged for $15 \mathrm{~min}$ at $7000 \mathrm{xg}$ at $4{ }^{\circ} \mathrm{C}$. Supernatant was carefully collected and stored at $-80^{\circ} \mathrm{C}$ until further use. Protein content in all the samples was determined using the BCA kit (Pierce, Pittsburg, PA) with BSA as a standard.

\section{Multiplex Immunoassays}

Cerebellar lysates were shipped on dry ice and a quantibody cytokine array (QAM-CYT-5) comprising of 40 proteins was performed at Ray Biotech. The 40 different proteins in the array comprised of chemokines, cytokines and growth factors: bFGF, BLC, CD30L, Eotaxin, Eotaxin-2, Fas L, G-CSF, GM-CSF, ICAM-1, IFN $\gamma$, IL-10, IL-12p40, IL-13, IL-15, IL-17, IL-1 $\alpha$, IL-1 $\beta$, IL-2, IL-21, IL-3, IL-4, IL-5, IL-6, IL-7, KC, Leptin, LIX, M-CSF, MCP-1, MCP-5, MIG, MIP- $1 \alpha$, MIP- $1 \gamma$, PF-4, RANTES, TARC, TCA-3, TNF RI, TNF RII and TNF $\alpha$ respectively. Samples from both the control and MIA offspring at the different ages (P1, P7 $n=4$ each and P14, P30 $n=6$ each) were run. Values below or at limit of detection (LOD) were considered undetected. The following proteins were not detected at any time point or condition and were not considered further: CD30L, Eotaxin, G-CSF, IL-13, Leptin, MIG, RANTES, and TCA-3.

\section{Enzyme Linked Immunosorbent Assay (ELISA)}

Cerebellar lysates from P14 control and MIA offspring were diluted 1:1 with the diluent and assayed for TNF $\alpha$
(Invitrogen) by ELISA using the standard procedures described in the product information sheet of the kit. All samples were run in duplicates and curve fit to obtain concentration values from the eight standards on the standard curve.

\section{Isolation of Synaptosomes}

Purified synaptosomes were isolated using a discontinuous sucrose density gradient by differential centrifugation as described in our earlier study (Pendyala et al, 2012). To obtain enough protein, P14 cerebella from two animals were pooled and a total of nine litters were used.

\section{Western Blot Analysis}

SDS-PAGE electrophoresis was performed using Biorad gel system as previously described (Pendyala et al, 2012). For western blot analyses $10 \mu \mathrm{g}$ of lysate and $20 \mu \mathrm{g}$ of synaptosomes from the control and MIA offspring were loaded onto $4-12 \%$ gradient Tris-Glycine gels (Biorad) under reducing conditions. Following transfer, blots were blocked using 5\% nonfat dried milk for $1 \mathrm{~h}$ at room temperature to minimize non-specific antibody binding. Immunoblotting was carried out with antibodies against TNFR1 $(1: 250$, Biovision), Cerebellin-1 (1:250, Abcam), GluR $\delta 2$ (1:2500, Millipore) and GAPDH $\left(1: 5000\right.$, Millipore) overnight at $4{ }^{\circ} \mathrm{C}$ with gentle rocking. Blots were washed thrice with TBS-Tween $0.1 \%$ followed by treatment with secondary antibody $(1: 2500$, $1: 2000$ and $1: 5000 \mathrm{HRP}$ conjugated anti rabbit IgG for TNFR1, Cerebellin-1 and GluR 2 2 respectively; $1: 5000$ HRP conjugated anti chicken for GAPDH) for 1 hour at room temperature. Blots were further washed thrice with TBS-Tween $0.1 \%$ and developed with $1: 1$ solution of Super Signal West Pico Chemiluminescent Substrate and Luminol/ Enhancer (Thermo Fisher Scientific, Rockford, IL, USA) and visualized using FluorHD2 imaging system (Cell Biosciences, Santa Clara, CA). Densitometric scanning was performed using the Image J software from NIH.

\section{Mixed Cerebellar Cultures}

Cerebella from postnatal day 0 mice were isolated and mixed cerebellar cultures were prepared using a protocol modified from (Baptista et al, 1994). Following dissociation, cells were plated in Basal Medium Eagle (BME; Sigma, B9638) supplemented with Sigma I-1884 and $10 \mathrm{mg} / \mathrm{ml} \mathrm{BSA}$ in 16 well Lab-Tek chamber slides (Nunc \#178599) coated with high MW poly-D lysine (Sigma, P7405), at 30000 cells/well. Cells were fixed at 14 days in vitro (DIV). A total of four control and four MIA litters were used for this study.

\section{Immunohistochemistry}

Fixed cells were incubated in $0.1 \%$ Triton-X for $2 \mathrm{~min}$, washed three times with PBS 1X, pre-incubated 1 hour in 5\% NGS with $0.1 \%$ Triton $100 \times$ in PBS and then immunostained with primary antibodies against GFP (polyclonal $1: 300$, Life Technologies), against VGluT1 (polyclonal $1: 300$, Millipore) and against GluR $\delta 2$ (monoclonal $1: 300$, Millipore) overnight. The secondary antibodies were Alexa 486,594 , and 647 coupled to goat anti-chicken, anti-guinea 
a

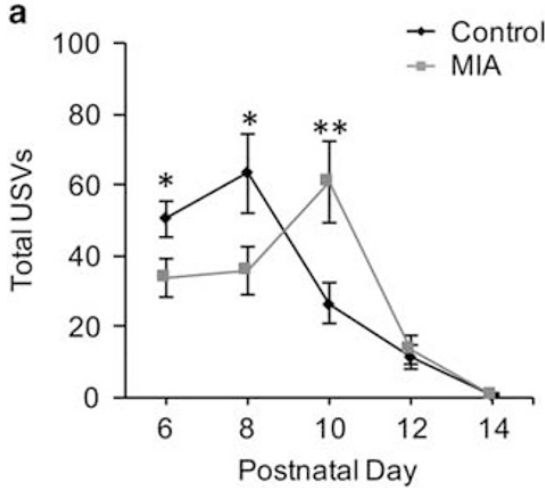

d

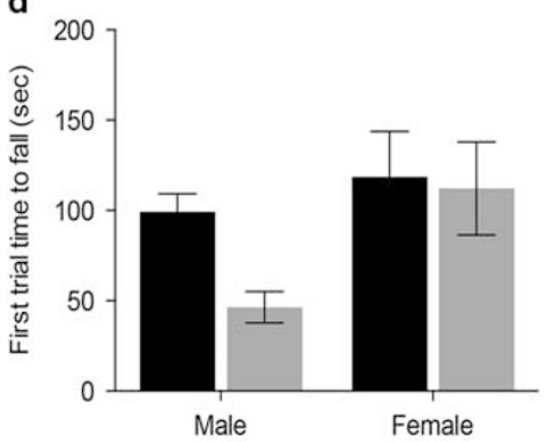

b

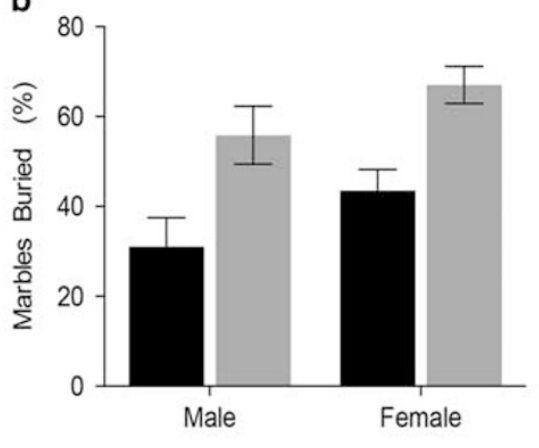

e

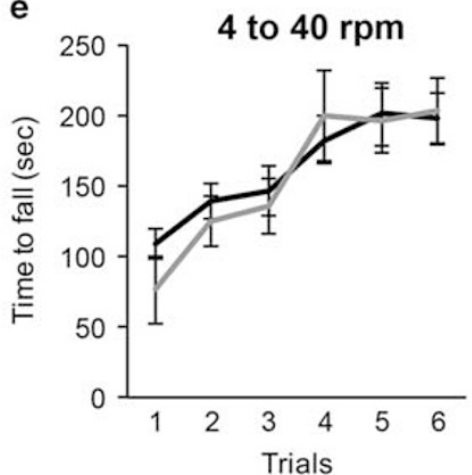

C

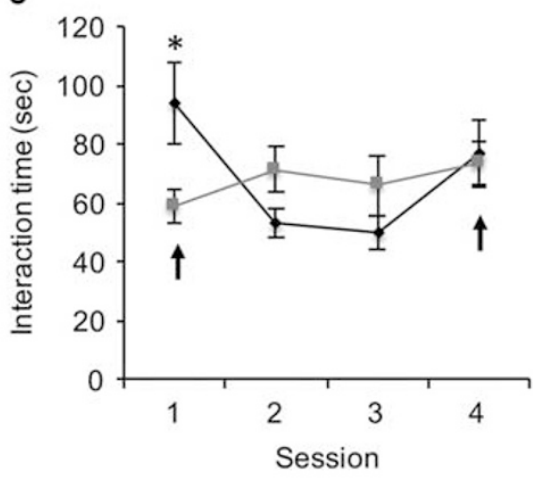

f

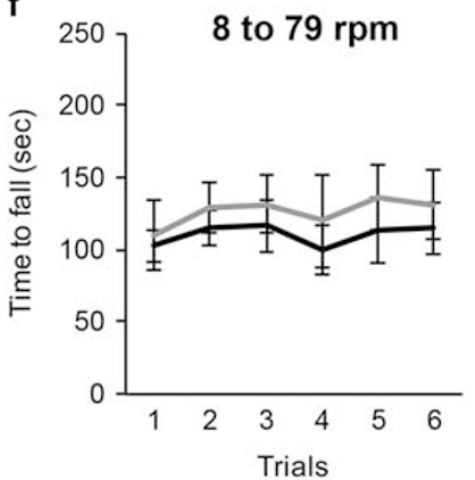

Figure I MIA offspring display ASD core behavioral deficits but not motor coordination deficits. (a) MIA offspring generated distinct patterns of total vocalizations. There was no significant main effect of $\operatorname{sex} F_{1,46}=0.006, P=0.94$. There was a significant main effect of postnatal days, $F_{4,90}=48.340, P<0.00$ I, and a significant postnatal day $\times$ condition interaction, $F_{4,90}=2.718, P=0.035$. Examinations of individual postnatal days using one-way ANOVA indicated that compared to control offspring, MIA offspring emitted a significantly lower number of USVs on postnatal days 6 and $8, F_{1,75}=5.560, P=0.021$ and $F_{1,75}=4.988, P=0.029$, respectively. MIA offspring emitted significantly more vocalizations on postnatal day $10 F_{1,75}=7.665, P=0.007$, as determined by oneway ANOVA based on postnatal day $x$ condition interaction. (b) P40 MIA and control offspring were assessed for repetitive behavior by marble burying assay. MIA offspring (male, $n=17$ and female, $n=18$ ) displayed higher marble burying than control offspring male $(n=16)$ and female $(n=18)$ with main effects of $\operatorname{sex}\left(F_{1,65}=4.7, P=0.03\right)$ and prenatal condition $\left(F_{I, 65}=19.59, P<0.000 I\right)$ but no significant sex $x$ prenatal condition interaction $\left(F_{1,65}=0.0138, P=0.9\right)$. $(c)$ MIA offspring display abnormal social interaction behaviors. Adult MIA $(n=9)$ and control $(n=9)$ offspring were repeatedly paired with the same partner for 3 sessions and a different partner in a fourth session. Arrows point to sessions with novel mouse pairing. There was a significant session $\times$ prenatal condition interaction, $F_{3,14}=5.144, P=0.013$. Post hoc one way ANOVA comparisons of individual sessions showed that the control group had a significantly longer interaction time compared to the MIA group during session I, $F_{1,16}=5.353, P=0.034$. In the control mice there was no significance between session I and 4 , but there was a significant decrease in the amount of time spent interacting from session I to $2, t(8)=3.45 \mathrm{I}, P=0.009$. MIA offspring showed an increase in interaction across sessions, with session 4 showing a significant increase in social behavior compared to session I, $t(8)=2.519, P=0.036$. (d) Females showed better motor coordination and there was a significant main effect of $\operatorname{sex}\left(F_{1}, 40=5.268, P=0.027\right)$. The main prenatal condition effect was not significant, $F_{1,40}=2.552, P=0.118$, nor the sex $\times$ prenatal condition interaction, $F(I, 40)=1.575, P=0.217$. (e and $f$ ) To determine if MIA offspring display increased motor learning, mice were tested on additional trials at 4-40 r.p.m. and 8-79 r.p.m. There was no main effect of sex $\left(F_{1.40}=2.135, P=0.152\right)$ therefore both sexes were pooled. Although there was a significant main effect of trials for both the $4-40$ r.p.m. trials $\left(F_{5,}, 210=29, P<0.000 \mathrm{I}\right)$ and the $8-79$ r.p.m. trials $\left(F_{5,210}=2.691, P=0.22\right)$, there was no significant main effect of prenatal condition $\left(F_{1,42}=0.1479, P=0.702\right)$ and $\left(F_{1,42}=1.391, P=0.24\right)$ for the $4-40$ and the 8-79 r.p.m. trials respectively as determined by RM two-way ANOVA. $* p<0.05, * * p<0.01$.

pig, anti-mouse, respectively ( $1: 500$, Invitrogen), in $1 \%$ NGS only in PBS.

\section{Confocal Imaging and Spine Analysis}

Confocal imaging was performed on a Zeiss, LSM 700 using a 40x 1.4 N.A Oil lens. Images of Purkinje cell dendrites were collected at $512 \times 512$ pixels with pixel size of $0.12 \mu \mathrm{m}$ and $\mathrm{Z}$ step of $0.21 \mu \mathrm{m}$, at 8 bit with $488 \mathrm{~nm}$ and 555, $659 \mathrm{~nm}$ lasers. Three-six Purkinje cells were imaged in each culture and a total of approximately 15 microns of a distal dendrite was analyzed per cell. Qualitative analysis of colocalization between GluR82 or GluR82/VGluT1 and dendritic spines was performed only on clearly visible spines that were protruding laterally from the dendrites using the ImarisColoc software as previously described (Coiro et al, 2015).

\section{Statistics}

Data are reported as mean \pm s.e.m. Data from USV, social interaction and the rotarod were analyzed using repeated measures ANOVA for within subject effects. Other analyses were done either using two-sided unpaired Student's $t$-test or with one- or two-way ANOVA with the Bonferonni method for post hoc multiple comparisons. Data was analyzed using the Graph Pad Prism software.

\section{RESULTS}

The goal of the study was to determine whether MIA induces changes in levels of cytokines in the cerebellum and whether these changes were accompanied by alterations in synapses. As we were interested in studying cerebellar Purkinje neuron 
synapses we have used a transgenic mouse that expresses EGFP under the Purkinje cell promoter PCP2 (also known as L7) on the FVB background. Since different mouse strains have different basal levels of behavior (Yang et al, 2013) and MIA can have differential effects depending on mouse strains (Schwartzer et al, 2013), we first tested if the MIA inducing protocol of a single injection of $20 \mathrm{mg} / \mathrm{kg}$ poly(I:C) at (E) 12.5 results in previously described behavioral impairments in the offspring.

\section{MIA Offspring Display Behavioral Deficits}

Previous studies demonstrated that MIA offspring have impairments in ultrasonic vocalizations (USV), marble burying and social interactions, which model the core behavioral deficits in ASD (Choi et al, 2016; Malkova et al, 2012). Measurement of USVs is a standard behavioral test to assess early communicative behavior. Pups were separated from mothers and the total number of USVs emitted by individual pups over a 3-min period on postnatal days $6,8,10$, 12 , and 14 were measured (Figure 1a). For the mice for which the sex was noted, there was no significant main effect of sex (Supplementary Figure 1A), we therefore pooled the male and female mice. Compared to controls $(n=37)$, MIA offspring $(n=38)$ emitted significantly fewer vocalizations on postnatal day 6 and $8(P=0.02$ and $P=0.029$, respectively). MIA offspring also produced a significantly higher number of USVs compared to controls on postnatal day $10(P=0.007)$ suggesting there is a shift in progression of the USVs in the MIA offspring. These results suggest that maternal separationinduced USV productions are altered in MIA offspring. Subsequent analysis at postnatal days 12 and 14 revealed no significant differences between the two groups thereby suggesting that MIA offspring display alterations in effective communication during the early stages of development.

To determine if MIA offspring display altered behaviors beyond early development, we tested them on the marble burying task that is a widely used test of repetitive and perseverative behaviors. At postnatal day 40, there was an 80 and $54 \%$ increase in marble burying of MIA males and females, respectively (Figure $1 \mathrm{~b}, P<0.0001$ ). Thus in the FVB mouse strain MIA results in increased repetitive behavior.

We also assessed whether MIA offspring have deficits in social interaction, assessing the active interaction time in a test mouse with a novel mouse over $5 \mathrm{~min}$. Postnatal day 90 mice from both the groups were tested for social interactions that spanned over four sessions (Figure 1c). There was no significant main effect of sex, we therefore pooled male and female mice (Supplementary Figure 1B). Compared to controls, MIA offspring displayed significantly less social interaction behavior in the first session $(P=0.034)$. Examination of the control group alone showed that there was no significance between session 1 and 4, both consisting of interactions with novel partners. However, there was a significant decrease in the amount of time spent interacting from session 1 to $2,(P<0.0001)$. In contrast, analysis of the MIA group alone showed a gradual increase in the amount of time spent interacting with partners across sessions, with session 4 showing a significant increase in social behavior compared to session $1(P<0.036)$. These results suggest that social interaction behaviors are altered in MIA offspring.
Altogether, these data demonstrate that MIA induced by a single injection of $20 \mathrm{mg} / \mathrm{kg}$ Poly(I:C) at E12.5 in the FVB strain results in offspring exhibiting similar behavioral impairments previously described in other strains.

Considering our focus on the cerebellum in this study we asked if motor coordination, a cerebellar-dependent behavior, was impaired in the MIA offspring. To measure motor coordination we compared the time to fall from an accelerating rotarod (4-40 r.p.m. over $300 \mathrm{~s}$ ) during the first trial. Females had better motor coordination than males as measured by time to fall on the first trial $(P=0.027)$. Despite a trend towards reduced motor coordination in male MIA offspring, MIA had no effect on motor coordination $(P=0.118$, Figure 1d). Recently, a new behavioral test has been used to measure acquired repetitive behaviors using the rotarod (Rothwell et al, 2014). In this paradigm, following the initial tests with rotation speed of 4-40 r.p.m. (Figure 1e and Supplementary Figure 1C), mice are switched to a more challenging phase for six sessions with the rotation speed at 8-79 r.p.m. over $300 \mathrm{~s}$ (Figure 1f). It has been shown that mice with mutations in NL3, a gene implicated in ASD, had enhanced learning at the higher speed compared to WT mice (Rothwell et al, 2014). Considering the increased marble burying behavior observed in MIA offspring we tested whether increased learning on the rotarod is also observed in MIA offspring. No difference in motor learning between MIA and control mice was observed in the 4-40 r.p.m. trials $(P=0.7)$ or in the $8-79$ r.p.m. trials $(P=0.244)$. These results suggest that MIA offspring do not have enhanced learning as measured by the rotarod assay.

\section{Poly(I:C) Treatment Induces a Neuroinflammatory State in the Cerebellum of MIA Offspring}

Previous studies determined that MIA results in altered levels of cytokines in the brain but the cerebellum has not been examined (Garay et al, 2013). To assess if MIA affects expression of chemokines and cytokines in the cerebellum during development, we performed a multiplex ELISA on the cerebellar lysates from control and MIA offspring at postnatal days 1, 7, 14, and 30. The multiplex ELISA comprised of a panel of 40 chemokines, cytokines and growth factors. We first determined the developmental expression profile in the cerebellum (Figure 2, Supplementary Table 1). Although most of these molecules were detected in the control cerebellum with concentrations ranging from $1-300 \mathrm{pg} / \mathrm{ml}$, we did observe higher concentrations ( $>1000 \mathrm{pg} / \mathrm{ml}$ ) of certain immune molecules such as IL-17 and PF-4 at several time points. While the expression levels of some proteins seemed relatively constant over development (ie, IL-1b, IL-2, IL-5, and TNFRI) others seemed to be more dynamically expressed. For example, M-CSF was not detected at P0 but was detected from P7 onwards. bFGF, IL-7, TNFRII and TNF $\alpha$, were detected at P1 and P30 but not at P7 and P14. Taken together, these data indicate that immune molecules are expressed in the developing cerebellum in an age-dependent manner.

We next compared how the levels of chemokines and cytokines are altered by MIA. We found a differential expression of several of the chemokines and cytokines between the two groups at different stages of development (Figure 3, Supplementary Table 2). At P1, two cytokines 

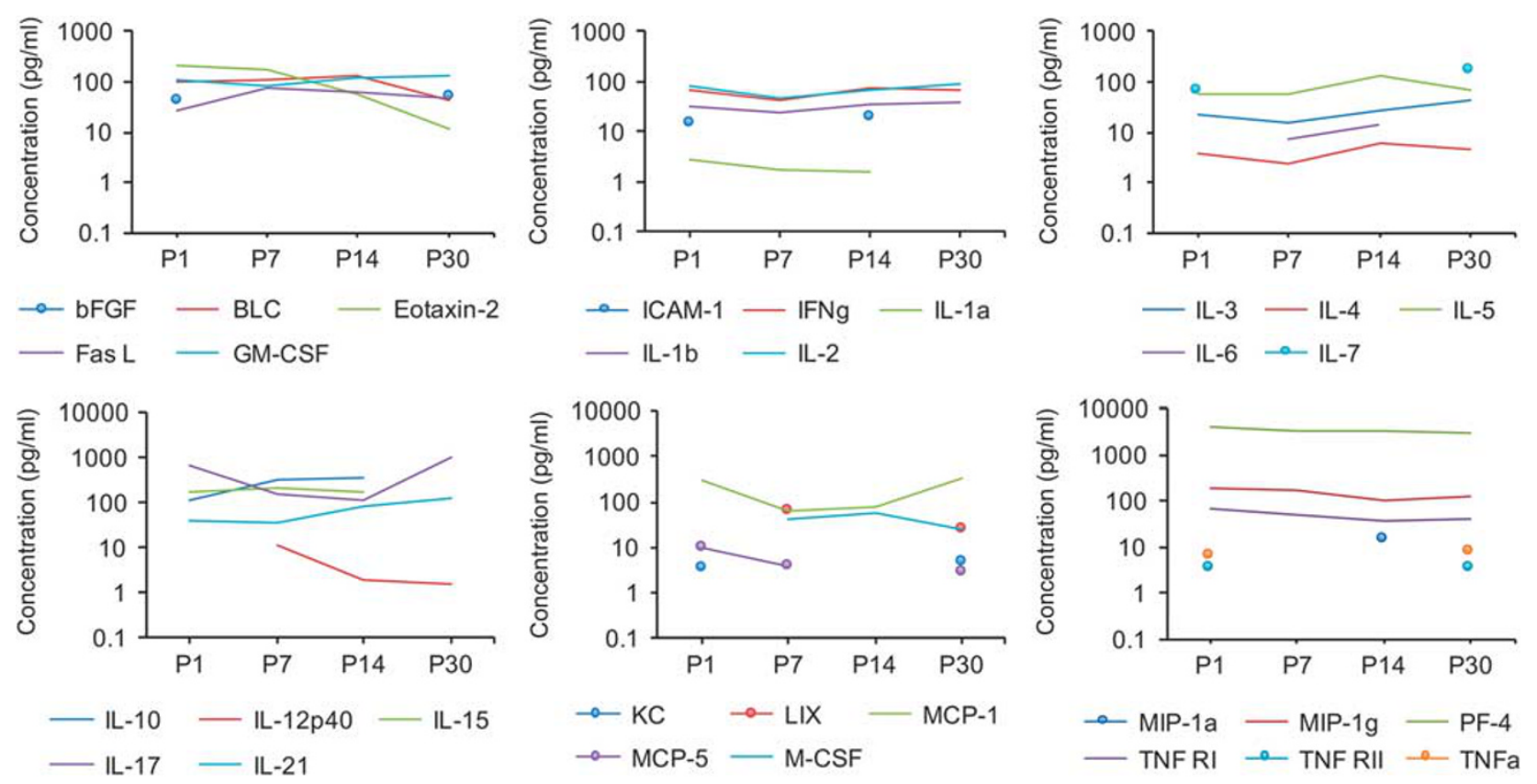

Figure 2 Dynamic expression of cytokines and chemokines in the cerebellum throughout normal development. To illustrate changes in cytokine concentrations with age, the average values for each cytokine concentration in $\mathrm{pg} / \mathrm{ml}$ of cerebellar lysate are plotted at the four ages examined. Values of mean \pm SEM concentration are included in Supplementary Table I.

Fas-L (2.79-fold, $P=0.027)$ and IL-6 (2.35-fold, $P=0.031)$ were significantly upregulated while there was a strong trend towards a decrease in the growth factor bFGF (0.38-fold, $P=0.059)$. At P7, levels of IL-2 (2.56-fold, $P=0.002)$, IL-3 (2.49-fold, $P=0.002$ ) and TNFRI (1.47fold, $P=0.023$ ) were significantly upregulated while Eotaxin-2 was downregulated (0.62-fold, $P=0.027)$ in the MIA offspring. At P14, which represents a peak in synaptogenesis, we observed a significant increase in the levels of MIP- $1 \gamma$ (2.16-fold, $P=0.02)$, TNF RI (1.64-fold, $P=0.004)$ and its ligand TNF $\alpha$ (3.21-fold, $P=0.01$ ). Reduction in the levels of ICAM-1 (0.23-fold, $P=0.001)$ and IL-10 (0.24-fold, $P=0.022$ ) were also noted in the MIA offspring. At P30, we found a significant increase in MIP- $1 \gamma$ (1.8-fold, $P=0.029)$ and a trend towards a large increase in M-CSF (11-fold, $P=0.08)$ while IFN $\gamma(0.55$-fold, $P=0.003)$ and IL-17 (0.58-fold, $P=0.031$ ) were significantly downregulated in the MIA offspring. Taken together, these data imply that MIA induces altered cytokine and chemokine profiles in the cerebellum that persist throughout development (Figure $3 \mathrm{~b}$ ).

Since our main emphasis was to elucidate how a neuroinflammatory state impacts the synapse, and since P14 is when there is a peak in synaptogenesis, our subsequent studies focused on P14. We were particularly interested in two proteins that were identified in the array at P14: the tumor necrosis factor receptor 1 (TNFR1) and its ligand $\mathrm{TNF} \alpha$ because of their known roles in regulating synapses (Habbas et al, 2015; Rothwell et al, 2014). To further validate the preliminary observation, we performed a western blot analysis on the cerebellum. There was an increase in TNFR1 expression in the MIA offspring (Figure $4 \mathrm{a}$ and b control: $1 \pm 0.11, n=23$; MIA: $1.39 \pm 0.11, n=26$; $P=0.017$, unpaired $t$-test). In parallel, ELISA analysis on the cerebellar lysates revealed an increase of 1.43 fold in TNF $\alpha$ expression in the MIA offspring (Figure 4c, control: $0.99 \pm 0.07, n=22$ animals; MIA: $1.45 \pm 0.19, n=19$ animals; $P=0.029$, unpaired $t$-test). These results demonstrate that a proinflammatory cytokine with previously described role in synaptic modulation is upregulated in the cerebellum of MIA offspring.

\section{MIA Offspring Display Reduction in Cerebellar Synaptic Organizing Proteins}

We next investigated how alterations in proinflammatory cytokine levels impact the development of synapses. Cerebellar synaptosomes were isolated from control and MIA offspring at P14 and analyzed by western blots for expression of synaptic organizing protein cerebellin- 1 and its postsynaptic interacting protein glutamate receptor delta 2 (GluR82). We observed a 31\% decrease in cerebellin-1 expression in the MIA offspring (Figure 5a, control: $1 \pm 0.09$, $n=14$; MIA: $0.69 \pm 0.09, n=20 ; P=0.035$ unpaired $t$-test). and a $27 \%$ reduction in GluR82 expression in the MIA offspring (Figure 5b, control: $1 \pm 0.12, n=17$; MIA: $0.73 \pm 0.05, n=21$ animals; $P=0.033$ unpaired t-test). These data demonstrate altered expression levels of important cerebellar synaptic organizing proteins in the MIA offspring suggesting that synaptic structure is likely to be altered in the cerebellum.

\section{Maternal Immune Activation Decreases Glutamatergic Synapse Density on Purkinje Neurons From Newborn Offspring}

To determine if reduced levels of synaptic organizer proteins results in reduced number of synapses, as has been shown for cortical neurons (Coiro et al, 2015; Elmer et al, 2013), we 
a
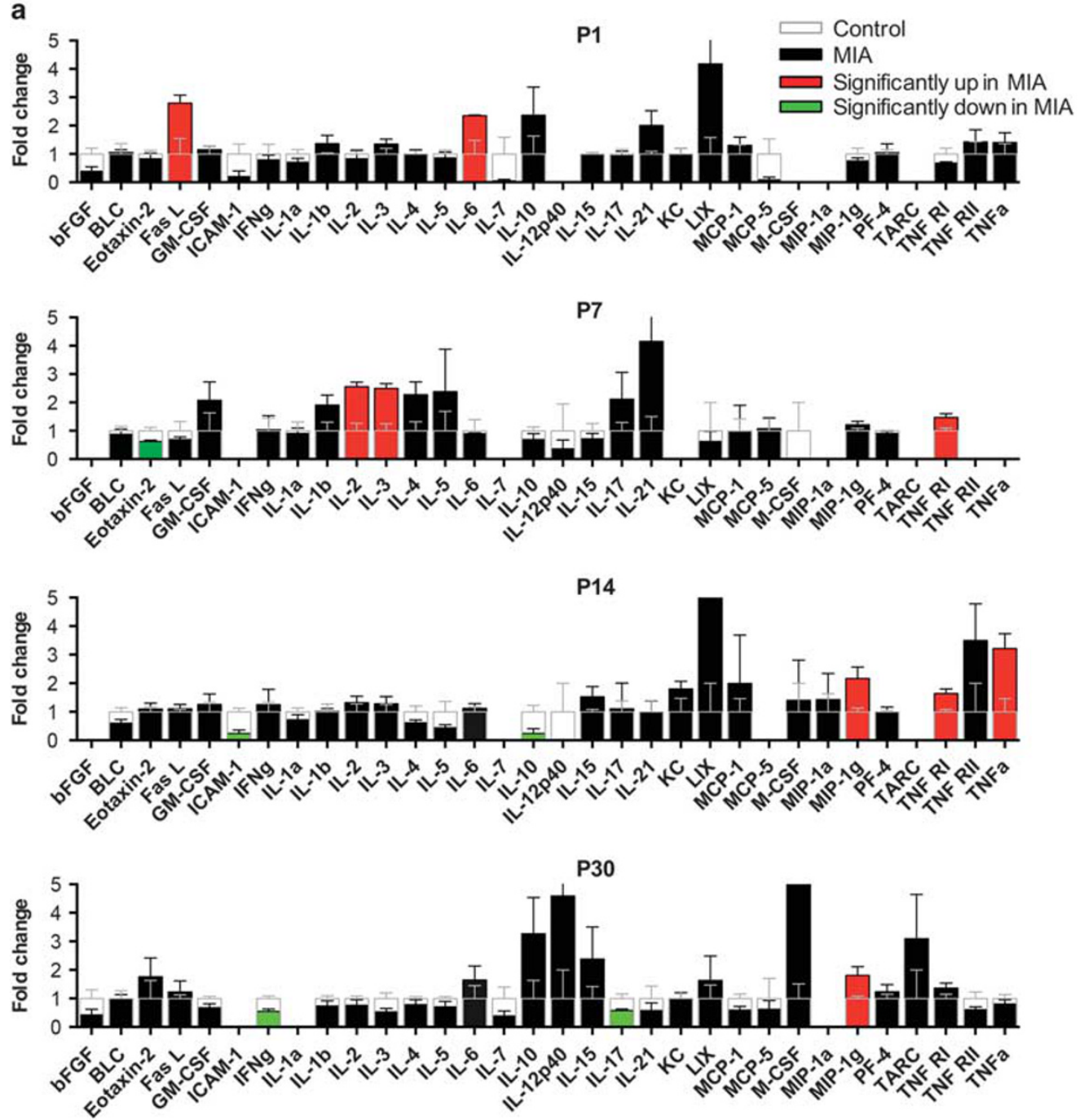

b

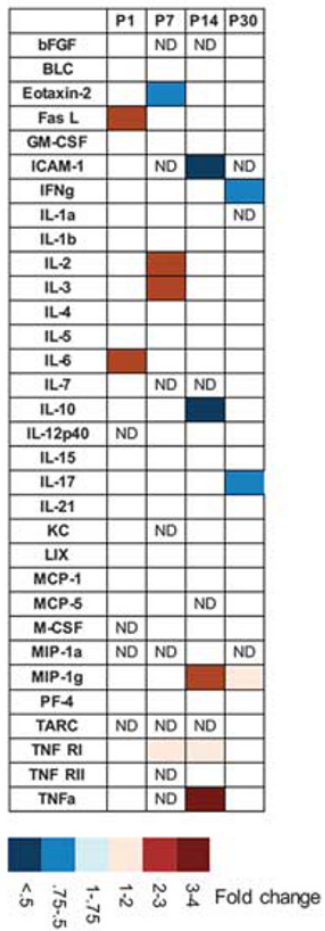

Figure 3 (a) MIA induces long lasting changes in cerebellar cytokines throughout development. Multiplex array showing the differential expression of various immune molecules in the cerebellar lysates of MIA offspring compared to control values at different stages of development. The average fold-change and SEM in cytokine concentration in cerebellum from MIA offspring compared to control values is plotted. Values from MIA offspring are either black, red, or green, and the control values are superimposed in clear bars. Red and green bars indicate statistically significant increases or decreases, respectively, compared to controls $(P<0.05)$. Increases are shown above I and decreases, below I. Values of fold change and SEMs are included in Supplementary Table 2. (b) A heat map is used to summarize the results for MIA-induced changes in cytokines and chemokines. The degree of change is indicated by the depth of color as indicated in the lower panel. ND = below the level of detection.

prepared dissociated mixed cerebellar cultures from PCP2GFP mice. Cells were immunostained for the presynaptic protein vesicular glutamate transporter-1 (VGluT1) and the postsynaptic density protein GluR $\delta 2$ (Figure 6a and b). Consistent with the reduced levels of GluR 22 in synaptosomes of MIA offspring, we have found that there was approximately a $10 \%$ reduction in proportion of dendritic spines that colocailzed with GluR $\delta 2$ puncta (control: $82.9 \pm 2.1 \%, n=19$ cells, MIA: $75.5 \pm 2.8 \%, n=17$ cells, $P=0.039$, Figure 6c). We next asked if reduced GluR $\delta 2$ in dendritic spines results in fewer synapses as identified as spines that contain GluR $\delta 2$ and are contacted by a VGluT1 puncta. In cultures from MIA offspring there was $15 \%$ reduction in the proportion of dendritic spines that colocolized with GluR82 and VGluT1 (control: 60.1 $\pm 3.1 \%$, $n=19$ cells, MIA: $51.4 \pm 3.6 \%, n=17$ cells, $P=0.035$, Figure 6d) indicating a reduction in number of synapses.
In summary, our study indicates that as previously described for the cortex, MIA results in altered expression of cytokines and impaired synaptic development in the cerebellum of the offspring, which might contribute to altered behavior in these mice.

\section{DISCUSSION}

Here we have investigated how the cerebellum is impacted by MIA using the poly(I:C) induction model. We report, that as previously described for other brain regions, in the cerebellum there are altered levels of proinflammatory cytokines during and after synaptogenesis that are accompanied by impairments in synaptic proteins and formation of synapses. These cerebellar changes are likely to contribute to the impairments in ASD relevant behaviors that these mice exhibit. 


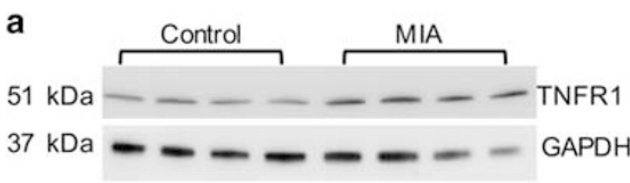

b
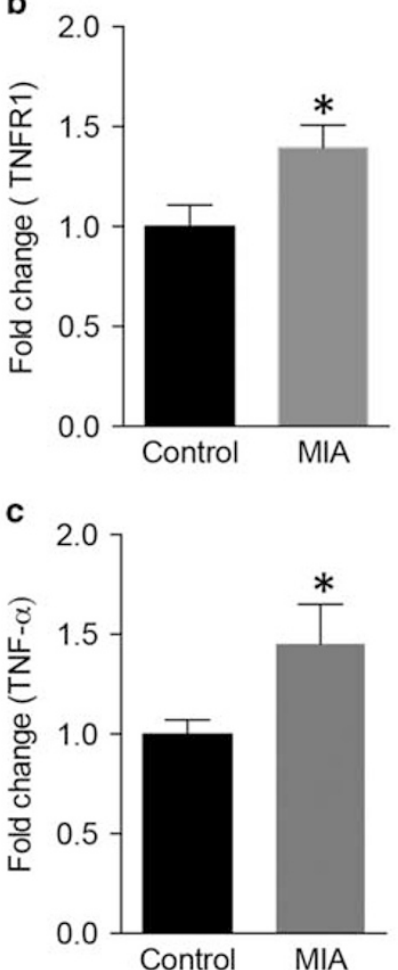

Figure 4 MIA induces increased expression of TNF $\alpha$ and TNFRI during synaptogenesis. (a) Western blot showing an increase in TNFRI expression in the cerebellar lysates of PI4 MIA offspring. (b) Bar graphs showing significant increase in TNFRI expression in MIA offspring $(n=23)$ versus controls $(n=26)$. (c) ELISA showing an increase in TNF $\alpha$ expression in the cerebellar lysates of MIA animals $(n=22)$ versus controls $(n=19)$. $* P<0.05$ as determined by an unpaired $t$-test.
Although the cerebellum is mainly known for its role in regulating motor control and balance, increasing evidence points to involvement in higher cognitive and affective functions (D'Mello and Stoodley, 2015). Based on connectivity and focal lesions studies it has been suggested that early cerebellar dysfunction may disrupt the maturation of distant neocortical circuits thus mediating some of the core ASD deficits (Wang et al, 2014). The cerebellum has been the most consistently observed site of pathology in ASD (Amaral et al, 2008; Courchesne et al, 1988; D'Mello and Stoodley, 2015; Levitt et al, 1999; Palmen et al, 2004; Wang et al, 2014) and functional cerebellar impairments were observed in both ASD and SZ (Allen and Courchesne, 2003; Brown et al, 2005; Pierce and Courchesne, 2001). In ASD, the cerebellum shows both gross and cellular defects, especially in the vermis with most notable histopathological feature being the reduction in the number of Purkinje neurons (Bailey et al, 1998; Kemper and Bauman, 1988). Interestingly, a similar reduction was reported in the number of Purkinje cells in the MIA offspring (Shi et al, 2009) and cerebellar impairments were reported in over 26 ASD mouse models (Ellegood et al, 2015).

It is becoming increasingly apparent that the causes of ASD and SZ are due to both genetic and environmental factors. Animal studies have shown that MIA can have different impact on different mouse strains suggesting important interactions between genetic variability and environmental insults. For example, while MIA led to an increase in USVs in the 8 day old BTBR mouse, no change was observed in the C57 mice at that age (Schwartzer et al, 2013). Our study is the first that has analyzed the impact of MIA on ASD-relevant behavior in the FVB strain. Our data show that MIA offspring display altered USVs with lower number of calls during postnatal days 6 and 8 but significantly higher number on days 10 . Although some studies previously shown reduced USVs in MIA offspring (Malkova et al, 2012) increased vocalization as we found in this study has been also reported in the second postnatal
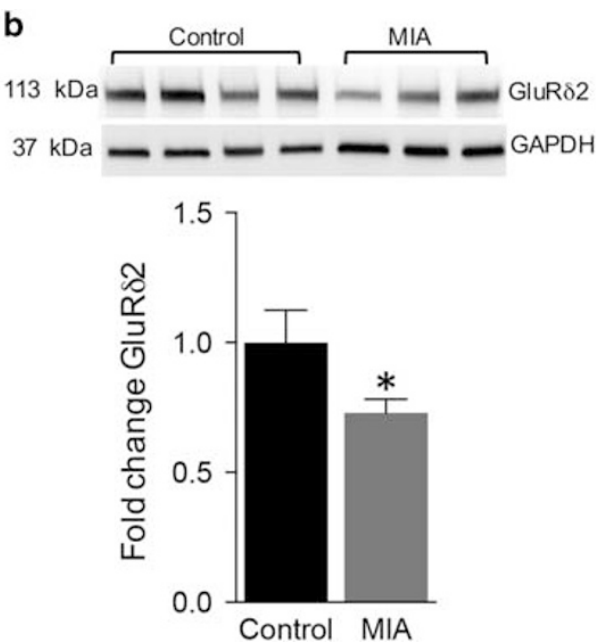

Figure 5 MIA induces a reduction in expression of synapse organizing proteins cerebellin-I and GluRס2. (a) Western blot showing a decrease in cerebellinI expression in the purified cerebellar synaptosomes of PI4 MIA offspring $(n=14)$ versus controls $(n=20)$. (b) Western blot showing a decrease in GluR 22 expression in the purified cerebellar synaptosomes of PI4 MIA animals $(n=17)$ versus controls $(n=21)$. Bar graphs for both the proteins are represented below each western blot. $* P<0.05$ as determined by an unpaired t-test. 

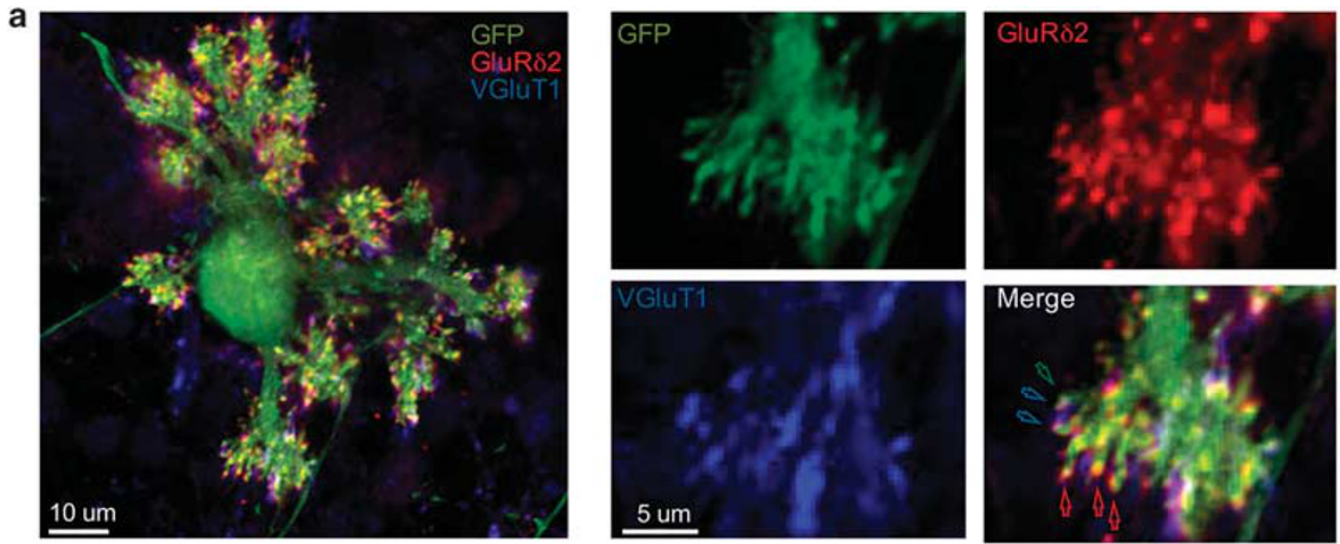

C
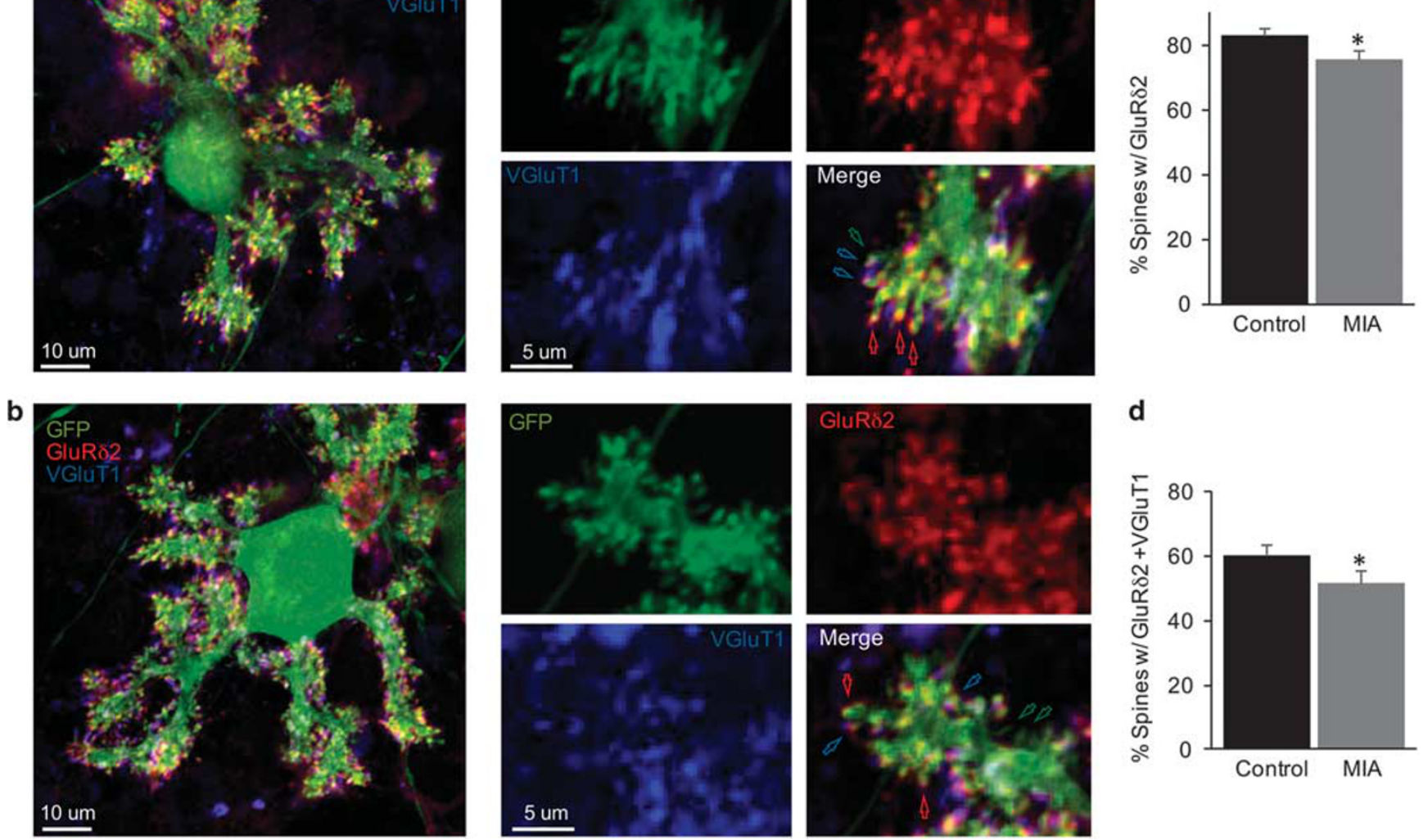

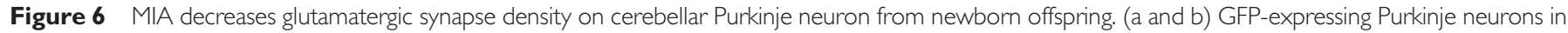

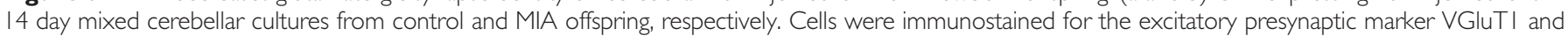

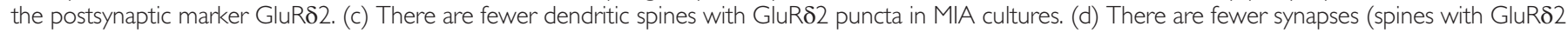

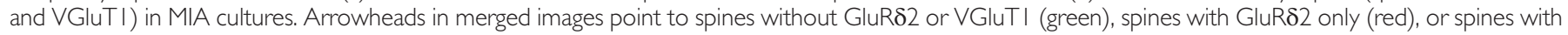
GluRס2 and VGluTI (blue). ${ }^{*} p<0.05$

week (Choi et al, 2016; Schwartzer et al, 2013). Previous studies implicate that the rat pup USVs is largely associated with negative affective states, as these calls are typically increased when pups are exposed to adverse environments such as maternal separation (Li et al, 2011) and cold temperature (Blumberg and Stolba, 1996). This is further supported by studies indicating that rat and mouse USVs may be reduced with the administration of anxiolytic drugs (Li et al, 2011; Nastiti et al, 1991). On the basis of these results, we may speculate that the affective development of MIA pups shows an altered trajectory. Isolation-induced USVs elicit pup retrieval by the parents suggesting that these calls play an important role in social communication between mother and infant (Branchi et al, 2004; D'Amato et al, 2005). An alternate theory regarding differences in the patterns of maternal separation-induced pup USVs is that MIA induces developmental abnormalities in communications (Bowers et al, 2013). Here, our analysis was limited to the number of USVs and further analysis could determine if different repertoire of isolation-induced USV subtypes are observed in MIA offspring as has been shown in other ASD mouse models (Scattoni et al, 2008).

We find that the behavioral deficits propagate into adulthood in these animals as evidenced by increased repetitive behavior and impairment in social interactions.
Interestingly, we have not observed significant changes in motor coordination in MIA offspring suggesting strain dependent effects of MIA on this behavior (Naviaux et al, 2014). We have also for the first time applied the modified accelerated rotarod test (Rothwell et al, 2014) to the MIA offspring. We have found no increased learning, which was previously ascribed to enhanced repetitive and stereotypic behavior. These data therefore suggest that increased marble burying and enhanced learning on the rotarod might be mediated by different circuits that are differentially affected by MIA.

Several studies have demonstrated altered expression of cytokines and markers of oxidative stress in biofluids such as blood, cerebrospinal fluid as well as the brain in ASD patients suggesting a neuroinflammatory condition to contribute to the symptoms and pathology of ASD (Ashwood et al, 2011a; Ashwood et al, 2011b; Chez et al, 2007; Molloy et al, 2006; Pardo et al, 2005; Vargas et al, 2005). Furthermore, these observations have been validated in rodent models especially the MIA model induced by the viral mimetic poly(I:C) (Garay et al, 2013; Patterson, 2009; Shi et al, 2009) although some studies failed to detect signs of inflammation in offspring born to immune-challenged mothers (Missault et al, 2014; Willi et al, 2013) and even showed reduction in inflammatory cytokines in the 
periphery (Pacheco-Lopez et al, 2013). To determine if poly (I:C) induced MIA leads to changes in the cerebellar cytokine milieu, we employed an unbiased multiplex ELISA platform to evaluate the changes in chemokine and cytokines expression in cerebellum during the first four weeks of postnatal development. Indeed a large number of chemokines and cytokines were differentially expressed suggesting that MIA leads to dynamic changes in the cytokine levels in the brains of the offspring and that these changes occur in an age-specific manner. Increased expression in the proinflammatory cytokine interlukin-6 (IL-6) in the cerebellum of ASD brains has been previously reported (Wei et al, 2011). Interestingly, we have found significant but transient increase in IL- 6 at postnatal day 1 . The early increase in IL-6 is thought to be critical for mediating the behavioral impairments in MIA offspring (Smith et al, 2007). The significant increase of IL-6 in the cerebellar lysates of MIA offspring at P1 could thus serve as a trigger eventually leading to an imbalance in the levels of other cytokines during the course of development. One such immune molecule increased in the MIA offspring later in development is the proinflammatory cytokine $\mathrm{TNF} \alpha$. Increased levels of TNF $\alpha$ has been found in both ASD (Chez et al, 2007; Vargas et al, 2005) and SZ (Miller et al, 2011) although reduction in $\mathrm{TNF} \alpha$ levels was also reported in SZ (Zhang et al, 2016). Impaired TNF $\alpha$ signaling has been implicated in impaired neurogenesis during maternal infections (Carpentier et al, 2011). Moreover, TNF $\alpha$ has been shown to be an important modulator of synaptic function and plasticity (Beattie et al, 2002) (Habbas et al, 2015). The altered expression of TNF $\alpha$ and TNFR1 is therefore also likely to affect synapses in the cerebellum. Our data complement a previous study that performed a similar analysis in the cortex and the hippocampus (Garay et al, 2013). Although some similarities exist; the antiinflammatory cytokine IL-10 is reduced in all brain regions examined, increase in IL-6 and TNF $\alpha$ was observed in the cerebellum while no change or opposite effect was observed in the cortical regions. Together these studies indicate that brain regional differences are observed with MIA. The cerebellar cytokine/chemokine profiles presented here provide evidence to the involvement of the cerebellum in MIA.

Dendritic spines have been shown to be impaired in various neuropsychiatric diseases, including in ASD and SZ brains (Glantz and Lewis, 2000; Hutsler et al, 2010; Penzes et al, 2011; Selemon and Goldman-Rakic, 1999). In the MIA mouse model, alterations in synaptic development have been previously demonstrated in the hippocampus (Ito et al, 2010) and the cortex (Coiro et al, 2015; Elmer et al, 2013) but not in the cerebellum of MIA offspring. Given the importance of immune proteins in normal synaptic development (Boulanger and Shatz, 2004) and the evidence of altered expression in cerebella of MIA offspring we tested if proteins with known functions in neuronal development and signaling to be dysregulated in the MIA offspring. Cerebellin 1, a protein that belongs to the $\mathrm{Clq}$ family, is a synaptic organizer that plays an essential role in the formation and maintenance of excitatory synapses in the cerebellum and interacts with the Purkinje cell specific glutamate receptor, GluR $\delta 2$, (Matsuda et al, 2010; Yuzaki, 2011). We found significant reduction in both cerebellin 1 and GluR $\delta 2$. Reduction in normal synapses and numerous naked spines lacking their presynaptic partners are found in mice with mutations in cerebellin 1 or GluR $\delta 2$ (Guastavino et al, 1990; Hirai et al, 2005; Kurihara et al, 1997). Consistent with their role as synaptic organizers we found that reduced levels of cerebellin 1 and GluR $\delta 2$ in MIA offspring is also associated with a deficit in the ability of cerebellar neurons to form synapses and an increased number of dendritic spines that are not in contact with a presynaptic terminal. Thus, MIA results in alterations in immune molecules and synaptic impairments throughout the brain, which are likely to contribute to the behavioral impairments observed in these mice.

\section{FUNDING AND DISCLOSURE}

This work was supported by: DOD/CDMRP Autism Research Program Idea Development Grant 13-1-0440 (AD), Nebraska Research Initiative (AD) and by National Institutes of Health grants R01MH107223 (AD) and P20RR018788. The authors declare no conflict of interest.

\section{ACKNOWLEDGMENTS}

We thank Collin Davis from UNL for his assistance with the USV and social interaction experiments. We would like to thank Mary Morison (Lycoming College) for assistance with the cerebellar cultures.

\section{AUTHOR CONTRIBUTIONS}

GP, SC, PC, ES, ML, and AD contributed to the study design and analyses of data. GP performed the biochemical analysis, YJ performed poly(I:C) injections, tissue culture preparation, immunohistochemistry and confocal imaging and rotarod experiments with RP, PC performed the synaptic image analysis. SC performed USV and social interactions tests while ES performed marble burying tests. GP wrote the first draft and $\mathrm{RP}, \mathrm{AD}$ participated in the subsequent drafts.

\section{REFERENCES}

Allen G, Courchesne E (2003). Differential effects of developmental cerebellar abnormality on cognitive and motor functions in the cerebellum: an fMRI study of autism. Am J Psychiatry 160: 262-273.

Amaral DG, Schumann CM, Nordahl CW (2008). Neuroanatomy of autism. Trends Neurosci 31: 137-145.

Ashwood P, Krakowiak P, Hertz-Picciotto I, Hansen R, Pessah I, Van de Water J (2011a). Elevated plasma cytokines in autism spectrum disorders provide evidence of immune dysfunction and are associated with impaired behavioral outcome. Brain Behav Immun 25: 40-45.

Ashwood P, Krakowiak P, Hertz-Picciotto I, Hansen R, Pessah IN, Van de Water J (2011b). Associations of impaired behaviors with elevated plasma chemokines in autism spectrum disorders. J Neuroimmunol 232: 196-199.

Atladottir HO, Thorsen P, Ostergaard L, Schendel DE, Lemcke S, Abdallah $\mathrm{M}$ et al (2010). Maternal infection requiring hospitalization during pregnancy and autism spectrum disorders. J Autism Dev Disord 40: 1423-1430.

Bailey A, Luthert P, Dean A, Harding B, Janota I, Montgomery M et al (1998). A full genome screen for autism with evidence for linkage to a region on chromosome 7q. Brain 121: 889-905. 
Baptista CA, Hatten ME, Blazeski R, Mason CA (1994). Cell-cell interactions influence survival and differentiation of purified Purkinje cells in vitro. Neuron 12: 243-260.

Bauman MD, Iosif AM, Smith SE, Bregere C, Amaral DG, Patterson $\mathrm{PH}$ (2014). Activation of the maternal immune system during pregnancy alters behavioral development of rhesus monkey offspring. Biol Psychiatry 75: 332-341.

Beattie EC, Stellwagen D, Morishita W, Bresnahan JC, Ha BK, Von Zastrow M et al (2002). Control of synaptic strength by glial TNFalpha. Science 295: 2282-2285.

Blumberg MS, Stolba MA (1996). Thermogenesis, myoclonic twitching, and ultrasonic vocalization in neonatal rats during moderate and extreme cold exposure. Behav Neurosci 110: 305-314.

Borrell J, Vela JM, Arevalo-Martin A, Molina-Holgado E, Guaza C (2002). Prenatal immune challenge disrupts sensorimotor gating in adult rats. Implications for the etiopathogenesis of schizophrenia. Neuropsychopharmacology 26: 204-215.

Boulanger LM, Shatz CJ (2004). Immune signalling in neural development, synaptic plasticity and disease. Nat Rev Neurosci 5: 521-531.

Bowers JM, Perez-Pouchoulen M, Edwards NS, McCarthy MM (2013). Foxp2 mediates sex differences in ultrasonic vocalization by rat pups and directs order of maternal retrieval. J Neurosci 33: 3276-3283.

Branchi I, Santucci D, Puopolo M, Alleva E (2004). Neonatal behaviors associated with ultrasonic vocalizations in mice (mus musculus): A slow-motion analysis. Dev Psychobiol 44: 37-44.

Brown SM, Kieffaber PD, Carroll CA, Vohs JL, Tracy JA, Shekhar A et al (2005). Eyeblink conditioning deficits indicate timing and cerebellar abnormalities in schizophrenia. Brain Cogn 58: 94-108.

Carpentier PA, Dingman AL, Palmer TD (2011). Placental TNFalpha signaling in illness-induced complications of pregnancy. Am J Pathol 178: 2802-2810.

Chez MG, Dowling T, Patel PB, Khanna P, Kominsky M (2007). Elevation of tumor necrosis factor-alpha in cerebrospinal fluid of autistic children. Pediatr Neurol 36: 361-365.

Choi GB, Yim YS, Wong H, Kim S, Kim H, Kim SV et al (2016). The maternal interleukin-17a pathway in mice promotes autismlike phenotypes in offspring. Science 351: 933-939.

Clancy B, Finlay BL, Darlington RB, Anand KJ (2007). Extrapolating brain development from experimental species to humans. Neurotoxicology 28: 931-937.

Coiro P, Padmashri R, Suresh A, Spartz E, Pendyala G, Chou S et al (2015). Impaired synaptic development in a maternal immune activation mouse model of neurodevelopmental disorders. Brain Behav Immun 50: 249-258.

Courchesne E, Yeung-Courchesne R, Press GA, Hesselink JR, Jernigan TL (1988). Hypoplasia of cerebellar vermal lobules VI and VII in autism. N Engl J Med 318: 1394-1354.

D'Amato FR, Scalera E, Sarli C, Moles A (2005). Pups call, mothers rush: does maternal responsiveness affect the amount of ultrasonic vocalizations in mouse pups? Behav Genet 35: 103-112.

D'Mello AM, Stoodley CJ (2015). Cerebro-cerebellar circuits in autism spectrum disorder. Front Neurosci 9: 408.

Ehninger D, Sano Y, de Vries PJ, Dies K, Franz D, Geschwind DH et al (2012). Gestational immune activation and Tsc2 haploinsufficiency cooperate to disrupt fetal survival and may perturb social behavior in adult mice. Mol Psychiatry 17: 62-70.

Ellegood J, Anagnostou E, Babineau BA, Crawley JN, Lin L, Genestine $\mathrm{M}$ et al (2015). Clustering autism: using neuroanatomical differences in 26 mouse models to gain insight into the heterogeneity. Mol Psychiatry 20: 118-125.

Elmer BM, Estes ML, Barrow SL, McAllister AK (2013). MHCI requires $\mathrm{MEF} 2$ transcription factors to negatively regulate synapse density during development and in disease. J Neurosci 33: 13791-13804.

Fatemi SH, Emamian ES, Sidwell RW, Kist DA, Stary JM, Earle JA et al (2002). Human influenza viral infection in utero alters glial fibrillary acidic protein immunoreactivity in the developing brains of neonatal mice. Mol Psychiatry 7: 633-640.

Garay PA, Hsiao EY, Patterson PH, McAllister AK (2013). Maternal immune activation causes age- and region-specific changes in brain cytokines in offspring throughout development. Brain Behav Immun 31: 54-68.

Glantz LA, Lewis DA (2000). Decreased dendritic spine density on prefrontal cortical pyramidal neurons in schizophrenia. Arch Gen Psychiatry 57: 65-73.

Guastavino JM, Sotelo C, Damez-Kinselle I (1990). Hot-foot murine mutation: behavioral effects and neuroanatomical alterations. Brain Res 523: 199-210.

Habbas S, Santello M, Becker D, Stubbe H, Zappia G, Liaudet N et al (2015). Neuroinflammatory TNFalpha impairs memory via astrocyte signaling. Cell 163: 1730-1741.

Hirai H, Pang Z, Bao D, Miyazaki T, Li L, Miura E et al (2005). Cbln1 is essential for synaptic integrity and plasticity in the cerebellum. Nat Neurosci 8: 1534-1541.

Hutsler JJ, Zhang H (2010). Increased dendritic spine densities on cortical projection neurons in autism spectrum disorders. Brain Res 1309: 83-94.

Ito HT, Smith SE, Hsiao E, Patterson PH (2010). Maternal immune activation alters nonspatial information processing in the hippocampus of the adult offspring. Brain Behav Immun 24: 930-941.

Kemper TL, Bauman M (1988). Neuropathology of infantile autism. J Neuropathol Exp Neurol 57: 645-652.

Knuesel I, Chicha L, Britschgi M, Schobel SA, Bodmer M, Hellings JA et al (2014). Maternal immune activation and abnormal brain development across CNS disorders. Nat Rev Neurol 10: 643-660.

Kurihara H, Hashimoto K, Kano M, Takayama C, Sakimura K, Mishina M et al (1997). Impaired parallel fiber-Purkinje cell synapse stabilization during cerebellar development of mutant mice lacking the glutamate receptor $\partial 2$ subunit. $J$ Neurosci 17: 9613-9623.

Levitt JG, Blanton R, Capetillo-Cunliffe L, Guthrie D, Toga A, McCracken JT (1999). Cerebellar vermis lobules VIII-X in autism. Prog Neuropsychopharm Bio Psych 23: 625-633.

Li M, He W, Heupel K (2011). Administration of clozapine to a mother rat potentiates pup ultrasonic vocalization in response to separation and re-separation: contrast with haloperidol. Behav Brain Res 222: 385-389.

Malkova NV, Yu CZ, Hsiao EY, Moore MJ, Patterson PH (2012). Maternal immune activation yields offspring displaying mouse versions of the three core symptoms of autism. Brain Behav Immun 26: 607-616.

Matsuda K, Miura E, Miyazaki T, Kakegawa W, Emi K, Narumi S et al (2010). Cbln1 is a ligand for an orphan glutamate receptor delta2, a bidirectional synapse organizer. Science 328: 363-368.

McAlonan GM, Li Q, Cheung C (2010). The timing and specificity of prenatal immune risk factors for autism modeled in the mouse and relevance to schizophrenia. Neurosignals 18: 129-139.

Meyer U, Nyffeler M, Engler A, Urwyler A, Schedlowski M, Knuesel I et al (2006). The time of prenatal immune challenge determines the specificity of inflammation-mediated brain and behavioral pathology. J Neurosci 26: 4752-4762.

Miller BJ, Buckley P, Seabolt W, Mellor A, Kirkpatrick B (2011). Meta-analysis of cytokine alterations in schizophrenia: clinical status and antipsychotic effects. Biol Psychiatry 70: 663-671.

Missault S, Van den Eynde K, Vanden Berghe W, Fransen E, Weeren A, Timmermans JP et al (2014). The risk for behavioural deficits is determined by the maternal immune response to prenatal immune challenge in a neurodevelopmental model. Brain Behav Immun 42: 138-146.

Molloy CA, Morrow AL, Meinzen-Derr J, Schleifer K, Dienger K, Manning-Courtney $\mathrm{P}$ et al (2006). Elevated cytokine levels in children with autism spectrum disorder. J Neuroimmunol 172: 198-205. 
Morgan JT, Chana G, Pardo CA, Achim C, Semendeferi K, Buckwalter J et al (2010). Microglial activation and increased microglial density observed in the dorsolateral prefrontal cortex in autism. Biol Psychiatry 68: 368-376.

Nastiti K, Benton D, Brain PF (1991). The effects of compounds acting at the benzodiazepine receptor complex on the ultrasonic calling of mouse pups. Behav Pharmacol 2: 121-128.

Naviaux JC, Schuchbauer MA, Li K, Wang L, Risbrough VB, Powell $\mathrm{SB}$ et al (2014). Reversal of autism-like behaviors and metabolism in adult mice with single-dose antipurinergic therapy. Transl Psychiatry 4: e400.

Onore CE, Schwartzer JJ, Careaga M, Berman RF, Ashwood P (2014). Maternal immune activation leads to activated inflammatory macrophages in offspring. Brain Behav Immun 38: 220-226.

Pacheco-Lopez G, Giovanoli S, Langhans W, Meyer U (2013). Priming of metabolic dysfunctions by prenatal immune activation in mice: relevance to schizophrenia. Schizophr Bull 39: 319-329.

Palmen SJ, van Engeland H, Hof PR, Schmitz C (2004). Neuropathological findings in autism. Brain 127(Pt 12): 2572-2583.

Pardo CA, Vargas DL, Zimmerman AW (2005). Immunity, neuroglia and neuroinflammation in autism. Int Rev Psychiatry 17: 485-495.

Patrich E, Piontkewitz Y, Peretz A, Weiner I, Attali B (2016). Maturation- and sex-sensitive depression of hippocampal excitatory transmission in a rat schizophrenia model. Brain Behav Immun 51: 240-251.

Patterson PH (2009). Immune involvement in schizophrenia and autism: etiology, pathology and animal models. Behav Brain Res 204: 313-321.

Patterson PH (2011). Maternal infection and immune involvement in autism. Trends Mol Med 17: 389-394.

Pendyala G, Buescher JL, Fox HS (2012). Methamphetamine and inflammatory cytokines increase neuronal $\mathrm{Na}(+) / \mathrm{K}(+)$-ATPase isoform 3: relevance for HIV associated neurocognitive disorders. PLOS ONE 7: e37604.

Penzes P, Cahill ME, Jones KA, VanLeeuwen JE, Woolfrey KM (2011). Dendritic spine pathology in neuropsychiatric disorders. Nat Neurosci 14: 285-293.

Pierce K, Courchesne E (2001). Evidence for a cerebellar role in reduced exploration and stereotyped behavior in autism. Biol Psychiatry 49: 655-664.

Rothwell PE, Fuccillo MV, Maxeiner S, Hayton SJ, Gokce O, Lim BK et al (2014). Autism-associated neuroligin-3 mutations commonly impair striatal circuits to boost repetitive behaviors. Cell 158: 198-212.

Sajdel-Sulkowska EM, Xu M, McGinnis W, Koibuchi N (2011). Brain region-specific changes in oxidative stress and neurotrophin levels in autism spectrum disorders (ASD). Cerebellum 10: 43-48.

Scattoni ML, Gandhy SU, Ricceri L, Crawley JN (2008). Unusual repertoire of vocalizations in the $\mathrm{BTBR} \mathrm{T}+\mathrm{tf} / \mathrm{J}$ mouse model of autism. PLoS ONE 3: e3067.
Schwartzer JJ, Careaga M, Onore CE, Rushakoff JA, Berman RF, Ashwood P (2013). Maternal immune activation and strain specific interactions in the development of autism-like behaviors in mice. Transl Psychiatry 3: e240.

Selemon LD, Goldman-Rakic PS (1999). The reduced neuropil hypothesis: a circuit based model of schizophrenia. Biol Psychiatry 45: 17-25.

Shi L, Fatemi SH, Sidwell RW, Patterson PH (2003). Maternal influenza infection causes marked behavioral and pharmacological changes in the offspring. J Neurosci 23: 297-302.

Shi L, Smith SE, Malkova N, Tse D, Su Y, Patterson PH (2009). Activation of the maternal immune system alters cerebellar development in the offspring. Brain Behav Immun 23: 116-123.

Smith SE, Li J, Garbett K, Mirnics K, Patterson PH (2007). Maternal immune activation alters fetal brain development through interleukin-6. J Neurosci 27: 10695-10702.

Vargas DL, Nascimbene C, Krishnan C, Zimmerman AW, Pardo CA (2005). Neuroglial activation and neuroinflammation in the brain of patients with autism. Ann Neurol 57: 67-81.

Wang SS, Kloth AD, Badura A (2014). The cerebellum, sensitive periods, and autism. Neuron 83: 518-532.

Wei H, Zou H, Sheikh AM, Malik M, Dobkin C, Brown WT et al (2011). IL-6 is increased in the cerebellum of autistic brain and alters neural cell adhesion, migration and synaptic formation. $J$ Neuroinflamm 8: 52.

Willi R, Harmeier A, Giovanoli S, Meyer U (2013). Altered GSK3beta signaling in an infection-based mouse model of developmental neuropsychiatric disease. Neuropharmacology 73: 56-65.

Yang M, Loureiro D, Kalikhman D, Crawley JN (2013). Male mice emit distinct ultrasonic vocalizations when the female leaves the social interaction arena. Front Behav Neurosci 7: 159.

Yuzaki M (2011). Cbln1 and its family proteins in synapse formation and maintenance. Curr Opin Neurobiol 21: 215-220.

Zhang XY, Tan YL, Chen DC, Tan SP, Yang FD, Wu HE et al (2016). Interaction of BDNF with cytokines in chronic schizophrenia. Brain Behav Immun 51: 169-175.

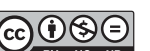

This work is licensed under a Creative Commons Attribution-NonCommercial-NoDerivs 4.0 International License. The images or other third party material in this article are included in the article's Creative Commons license, unless indicated otherwise in the credit line; if the material is not included under the Creative Commons license, users will need to obtain permission from the license holder to reproduce the material. To view a copy of this license, visit http://creativecommons.org/licenses/by-nc-nd/4.0/

(c) The Author(s) 2017

Supplementary Information accompanies the paper on the Neuropsychopharmacology website (http://www.nature.com/npp) 